\title{
Tissue specificity of insulin resistance in humans: fat in the liver rather than muscle is associated with features of the metabolic syndrome
}

\author{
A. Kotronen • A. Seppälä-Lindroos • R. Bergholm • \\ H. Yki-Järvinen
}

Received: 15 August 2007 / Accepted: 8 October 2007 / Published online: 16 November 2007

(C) Springer-Verlag 2007

\begin{abstract}
Aims/hypothesis The aim of this study was to investigate whether intrahepatic and intramyocellular fat are related to insulin resistance in these respective tissues or to the metabolic syndrome.

Methods Hepatic (insulin $1.8 \mathrm{pmol} \mathrm{kg}^{-1} \mathrm{~min}^{-1}$ combined with $\left[3{ }^{3} \mathrm{H}\right]$ glucose) and muscle (insulin $6.0 \mathrm{pmol} \mathrm{kg} \mathrm{km}^{-1}$ ) insulin sensitivity were measured on separate occasions in 45 non-diabetic men (age $42 \pm 1$ years, BMI $26.2 \pm 0.6 \mathrm{~kg} / \mathrm{m}^{2}$ ) using the euglycaemic-hyperinsulinaemic clamp. Liver fat and intramyocellular lipid (IMCL) were measured by proton magnetic resonance spectroscopy and body composition by magnetic resonance imaging. We also determined fasting serum insulin and adiponectin concentrations, components of the metabolic syndrome and maximal oxygen consumption.

Results In participants with high [median 12.0\% (interquartile range $5.7-18.5 \%)$ ] vs low [2.0\% (1.0-2.0\%)] liver fat, fasting serum triacylglycerols $(1.6 \pm 0.2$ vs $1.0 \pm$ $0.1 \mathrm{mmol} / 1, p=0.002)$ and fasting serum insulin ( $55 \pm 4 \mathrm{vs}$ $32 \pm 2 \mathrm{pmol} / \mathrm{l}, p<0.0001)$ were increased and serum HDLcholesterol $(1.26 \pm 0.1$ vs $1.48 \pm 0.1 \mathrm{mmol} / 1, p=0.02)$ and fasting serum adiponectin $(9.5 \pm 1.2 \mathrm{vs} 12.2 \pm 1.2 \mu \mathrm{g} / \mathrm{ml}, p=$ $0.05)$ decreased. In participants with high $[19.5 \%$ (16.0 $26.0 \%)]$ vs low [5.0\% (2.3-7.5\%)] IMCL, these parameters were comparable. Liver fat was higher in participants with

\footnotetext{
A. Kotronen $(\bowtie) \cdot$ A. Seppälä-Lindroos · R. Bergholm •

H. Yki-Järvinen

Department of Medicine, Division of Diabetes,

University of Helsinki,

P.O. Box 700, room C418B, FIN-00029 HUCH,

Helsinki, Finland

e-mail: anna.kotronen@helsinki.fi
}

A. Kotronen $\cdot$ R. Bergholm

Minerva Medical Research Institute,

Helsinki, Finland
\end{abstract}

$[10.5 \%(3.0-18.0 \%)]$ than in those without $[2.0 \%(1.5-$ $6.0 \%), p=0.010]$ the metabolic syndrome, even independently of obesity, while IMCL was comparable. Insulin suppression of glucose rate of appearance and serum NEFA was significantly impaired in the high liver fat group.

Conclusions/interpretation Fat accumulation in the liver rather than in skeletal muscle is associated with features of the metabolic syndrome, i.e. increased fasting serum triacylglycerols and decreased fasting serum HDL-cholesterol, as well as with hyperinsulinaemia and low adiponectin.

Keywords Hepatic insulin resistance .

Intramyocellular lipid · Liver fat · Metabolic syndrome .

Muscle insulin resistance $\cdot$ Non-esterified fatty acids

$\begin{array}{ll}\text { Abbreviations } \\ \text { ALT } & \text { alanine aminotransferase } \\ \text { AST } & \text { aspartate aminotransferase } \\ \text { FFM } & \text { fat free mass } \\ \text { IMCL } & \text { intramyocellular lipid } \\ \text { MRI } & \text { magnetic resonance imaging } \\ \text { ppm } & \text { parts per million } \\ R_{\mathrm{a}} & \text { rate of appearance } \\ R_{\mathrm{d}} & \text { rate of disappearance } \\ \dot{V} \mathrm{O}_{2 \text { max }} & \text { maximal oxygen consumption }\end{array}$

\section{Introduction}

The metabolic syndrome, as currently defined by the International Diabetes Federation, includes an increased waist circumference, elevated serum glucose, triacylglycerols and blood pressure, as well as a low HDL-cholesterol concentration [1]. Although insulin resistance is considered 
a core component of the syndrome [2], the contribution of insulin resistance in muscle, liver and other tissues, such as adipose tissue, to features of the metabolic syndrome is unclear. Insulin normally inhibits the production of both glucose and VLDL from the liver [3, 4]. When the liver is fatty, the ability of insulin to suppress glucose production is impaired [5, 6], as is the ability of insulin to suppress VLDL production while VLDL clearance is unaltered [7]. Insulin resistance in the liver can thus be expected to be accompanied by mild hyperglycaemia, hyperinsulinaemia, hypertriacylglycerolaemia and a low HDL-cholesterol concentration. Adiponectin is an insulin sensitiser, serum levels of which are low in obesity [8]. In mice, it acts exclusively in the liver to reduce steatosis and inflammation, and increase insulin sensitivity [8]. Serum adiponectin would therefore be expected to correlate with hepatic rather than muscle insulin sensitivity.

Intramyocellular lipid (IMCL) has been shown to be inversely [9-11], not at all [12] and positively [12] correlated with insulin sensitivity in muscle. The paradoxical positive correlation has been attributed to the 'exercise paradox', the propensity of trained athletes to have increased IMCL stores, which can be effectively utilised to sustain energy needs during prolonged exercise $[13,14]$. On the other hand, obesity is accompanied by an increase in IMCL $[12,15]$. Thus, while obesity and maximal oxygen consumption $\left(\dot{V} \mathrm{O}_{2 \max }\right)$ have opposite effects on muscle insulin sensitivity, they are both associated with increased IMCL.

In the present study, we hypothesised that hepatic fat accumulation is associated to a greater degree with features of the metabolic syndrome than is fat accumulation in the skeletal muscle. There are no human studies where insulin sensitivity was measured in the same participants in both the liver and skeletal muscle and related to intramyocellular and intrahepatic lipid contents or to the clinical measure of insulin resistance i.e. the metabolic syndrome. We studied 45 non-diabetic, apparently healthy men, in whom insulin sensitivity was measured on two separate occasions under conditions which were optimised to measure either muscle or hepatic insulin sensitivity. Intrahepatic and intramyocellular fat were determined in each participant using proton magnetic resonance spectroscopy, and subcutaneous and intra-abdominal fat were measured by magnetic resonance imaging (MRI). In addition, since physical fitness may influence intramyocellular fat in particular [12, 16, 17], $\dot{V} \mathrm{O}_{2 \max }$ was determined in each participant.

\section{Methods}

Participants and study design We recruited 45 men from occupational health services in Helsinki on the basis of the following inclusion criteria: (1) age 18-60 years; (2) no known acute or chronic disease, except for obesity, based on history, physical examination and standard laboratory tests (blood counts, serum creatinine, thyroid-stimulating hormone, electrolyte concentrations and ECG); (3) no regular medication; and (4) alcohol consumption $<20 \mathrm{~g}$ per day. Individuals with elevated liver enzymes [serum alanine aminotransferase (ALT), aspartate aminotransferase (AST) and gamma glutamyl transferase] were not excluded. The study protocol was approved by the Ethics Committee of the Helsinki University Central Hospital and each participant provided written informed consent. Data on liver fat and hepatic insulin sensitivity of 30 men have previously been reported [6].

The participants were studied on four separate occasions. All measurements were performed within 1 month. These measurements included: (1) hepatic insulin sensitivity; (2) liver fat and IMCL (by proton magnetic resonance spectroscopy), intra-abdominal and subcutaneous fat by MRI; (3) muscle insulin sensitivity; and (4) $\dot{V} \mathrm{O}_{2 \max }$.

Hepatic insulin sensitivity The dose-response characteristics of insulin suppression of glucose production and stimulation of glucose utilisation differ [18]. At insulin concentrations typically achieved during measurement of insulin sensitivity by the insulin clamp technique (insulin infusion rate $6.0 \mathrm{pmol} \mathrm{kg}^{-1} \mathrm{~min}^{-1}$ or $240 \mathrm{pmol} \mathrm{m}^{-2} \mathrm{~min}^{-1}$ ) [19], hepatic glucose production in normal individuals is completely suppressed $[18,20]$; therefore, any interindividual variation in hepatic insulin sensitivity is missed. To measure hepatic insulin sensitivity, a prolonged low-dose insulin infusion is required [21], and it is necessary to use a glucose tracer to distinguish between insulin effects on glucose production and utilisation [19, 21].

We chose an insulin infusion rate of $1.8 \mathrm{pmol} \mathrm{kg}^{-1} \mathrm{~min}^{-1}$ to maximise the possibility of detecting inter-individual differences in hepatic insulin sensitivity. At 0800 hours, after an overnight fast, two indwelling catheters were placed in an antecubital vein and retrogradely in a heated hand vein for infusion of glucose, insulin and $\left[3-{ }^{3} \mathrm{H}\right]$ glucose, and for sampling of arterialised venous blood. To determine rate of appearance $\left(R_{\mathrm{a}}\right)$ and rate of disappearance $\left(R_{\mathrm{d}}\right)$ under basal and hyperinsulinaemic conditions, $\left[3-{ }^{3} \mathrm{H}\right]$ glucose was infused in a primed $\left(74 \times 10^{4} \mathrm{~Bq}\right)$ continuous $\left(7.4 \times 10^{4} \mathrm{~Bq} / \mathrm{min}\right)$ fashion for a total of $360 \mathrm{~min}[5,19]$. Baseline blood samples were taken for measurement of fasting serum insulin and glucose concentrations, and for the biochemical measurements (serum ALT, serum AST, fasting serum triacylglycerols, fasting serum HDL concentrations, fasting serum C-peptide, fasting serum adiponectin and fasting serum NEFA). After $120 \mathrm{~min}$, insulin was infused in a primed continuous (1.8 $\mathrm{pmol} \mathrm{kg}^{-1} \mathrm{~min}^{-1}$ ) fashion as previously described [5]. Plasma glucose was maintained at $5 \mathrm{mmol} / \mathrm{l}(90 \mathrm{mg} / \mathrm{dl})$ until $360 \mathrm{~min}$ using a 
variable rate infusion of $20 \%$ glucose [19]. Blood samples for measurement of glucose specific activity and NEFA concentrations were taken at 90,100,110 and $120 \mathrm{~min}$ and at 15-30 min intervals between 120 and $360 \mathrm{~min}$. Serum free insulin concentrations were measured every $60 \mathrm{~min}$ during the insulin infusion.

Muscle insulin sensitivity The $6.0 \mathrm{pmol} \mathrm{kg}^{-1} \mathrm{~min}^{-1}$ insulin infusion rate was chosen because it stimulates glucose disposal half-maximally, which occurs mainly in skeletal muscle $[18,22]$ and completely suppresses hepatic glucose production in non-diabetic individuals $[18,20]$. The study was started at 0800 hours after a $12 \mathrm{~h}$ fast. Two 18 gauge catheters (Venflon; Viggo-Spectramed, Helsingborg, Sweden) were inserted, one in the left antecubital vein for infusions of insulin and glucose, and another retrogradely in an ipsilateral heated dorsal hand vein for withdrawal of arterialised venous blood. Insulin (Actrapid Human; Novo Nordisk, Copenhagen, Denmark) was infused in a primedcontinuous manner at a rate of $6.0 \mathrm{pmol} \mathrm{kg}{ }^{-1} \mathrm{~min}^{-1}$ for $120 \mathrm{~min}$. Normoglycaemia was maintained by adjusting the rate of a $20 \%$ glucose infusion based on plasma glucose measurements, which were performed every $5 \mathrm{~min}$ from arterialised venous blood. Blood samples for serum free insulin concentrations were withdrawn every $30 \mathrm{~min}$ during the $2 \mathrm{~h}$ insulin infusion. The $M$ value (0-120 min) was calculated from the glucose infusion rate after correction for changes in the glucose pool size and expressed per fat free mass (FFM).

Liver fat content (proton magnetic resonance spectroscopy) Localised single voxel $\left(2 \times 2 \times 2 \mathrm{~cm}^{3}\right)$ proton spectra were acquired using a 1.5-T whole-body system (Magnetom Vision; Siemens, Erlangen, Germany), which consisted of a combination of whole-body and loop surface coils for radiofrequency transmitting and signal receiving. T1-weighted high-resolution MRI scans were used for localisation of the voxel of interest within the right lobe of the liver. Magnetic resonance spectroscopy measurements of liver fat were performed in the middle of the right lobe of the liver at a location that was individually determined for each participant; vascular structures and subcutaneous fat tissue were avoided when selecting the voxel. Participants lay on their stomachs on the surface coil, which was embedded in a mattress in order to minimise abdominal movement due to breathing. The single voxel spectra were recorded using the stimulated-echo acquisition mode sequence, with an echo time of $20 \mathrm{~ms}$, a repetition time of $3,000 \mathrm{~ms}$, a mixing time of $30 \mathrm{~ms}$ and 1,024 data points over $1000 \mathrm{kHz}$ spectral width with 32 averages. Water-suppressed spectra with 128 averages were also recorded to detect weak lipid signals. A short echo time and long repetition time were chosen to ensure a fully relaxed water signal, which was used as an internal standard. Chemical shifts were measured relative to water at 4.80 [parts per million (ppm)]. The methylene signal, which represents intracellular triacylglycerol, was measured at $1.4 \mathrm{ppm}$. Signal intensities were quantified by using an analysis program, VAPRO-MRUI (http://www. mrui.uab.es/mrui/, last accessed in October 2007). Spectroscopic intracellular triacylglycerol content (liver fat) was expressed as a ratio of the area under the methylene peak to that under the methylene and water peaks $(\times 100=$ liver fat $\%)$. This measurement has been validated against histologically determined lipid content [23] and against estimates of fatty degeneration or infiltration by X-ray computer-assisted tomography [5]. All spectra were analysed by physicists who were unaware of any of the clinical data.

IMCL (proton magnetic resonance spectroscopy) Magnetic resonance images for localisation and proton magnetic resonance spectroscopy were acquired using a $1.5 \mathrm{~T}$ system (Magnetom Vision; Siemens) as previously described [24]. A loop surface coil was used for detection. The volunteers lay supine, with the left leg immobilised by firm padding. One third of the distance from the trochanter major of the femur to the middle of the patella was measured and the centre of the coil was placed in contact with that spot. The ${ }^{1} \mathrm{H}$-spectra were obtained from quadriceps femoris (vastus lateralis) muscle. The volume of interest, voxel $(13 \times 13 \times$ $20 \mathrm{~mm}^{3}$ ), was placed inside the lateral part of the vastus lateralis muscle to ensure parallel fibre orientation. The position of the voxel was chosen on the $T_{1}$-weighted magnetic resonance images so that it did not contain any visible fat or fasciae that are known to contain significant amounts of adipocytes [25] and would affect the resonance-frequency shift data. Spatial localisation was achieved by using a stimulated echo acquisition mode applied with a repetition time of $3,000 \mathrm{~ms}$, with an echo time of $20 \mathrm{~ms}$ and a mixing time of $30 \mathrm{~ms}$. We used 128 excitations with water presaturation. The resonance at $1.5 \mathrm{ppm}$ originates from the extramyocellular $\mathrm{CH}_{2}$ protons of lipids (triacylglycerols and fatty acids) and the resonance at $1.3 \mathrm{ppm}$ from intramyocellular $\mathrm{CH}_{2}$ protons of lipids [25-27]. Resonance intensities were related to resonance at $3.0 \mathrm{ppm}$, which is derived from the $\mathrm{CH}_{3}$ protons of total creatine (creatine and phosphocreatine). IMCL was expressed as a ratio of the area under the intramyocellular methylene peak to that under the methylene and total creatine peaks $(\times 100=\mathrm{IMCL} \%)$. The spectral data were fitted in time domain with the program MRUI, based on the solving of nonlinear least squares problems [26].

Intra-abdominal fat and subcutaneous fat (MRI) A series of 16 T1-weighted trans-axial scans were acquired from a region extending from $8 \mathrm{~cm}$ above to $8 \mathrm{~cm}$ below the fourth and fifth lumbar interspace (16 slices, field of view $375 \times$ 
$500 \mathrm{~mm}^{2}$, slice thickness $10 \mathrm{~mm}$, breath-hold repetition time $138.9 \mathrm{~ms}$, echo time $4.1 \mathrm{~ms}$ ). Intra-abdominal and subcutaneous fat areas were measured using an image analysis program (Alice 3.0; Parexel, Waltham, MA, USA). A histogram of pixel intensity in the intra-abdominal region was displayed, and the intensity corresponding to the nadir between the lean and fat peaks was used as a cut-off point. Intra-abdominal adipose tissue was defined as the area of pixels in the intra-abdominal region above this cutoff point. For calculation of subcutaneous adipose tissue area, a region of interest was first drawn manually at the demarcation of subcutaneous adipose tissue and intra-abdominal adipose tissue, as previously described [5].

Maximal aerobic power Maximal aerobic power $\left(\dot{V} \mathrm{O}_{2 \max }\right)$ was measured directly using an incremental work-conducted upright exercise test with an electrically braked cycle ergometer (Ergometer Ergoline 900ERG; Ergoline, Bitz, Germany) combined with continuous analysis of expiratory gases and minute ventilation $\left(\mathrm{V}_{\max } 229\right.$ series; SensorMedics, Yorba Linda, CA, USA). Exercise was started at a work load of 50 watts. The work load was then increased by 50 watts every 3 min until perceived exhaustion or a respiratory quotient of 1.10 was reached. Maximal aerobic power was defined as the $\dot{V} \mathrm{O}_{2 \max }$ during the last 30 s of exercise.

Diagnosis of the metabolic syndrome The metabolic syndrome was defined according to criteria of the International Diabetes Federation [1]: central obesity (waist circumference $\geq 94 \mathrm{~cm}$ in men and $\geq 80 \mathrm{~cm}$ in women) and at least two of the following factors: (1) serum triacylglycerols $\geq 1.70 \mathrm{mmol} / \mathrm{l}$ or specific treatment for this lipid abnormality; (2) serum HDL-cholesterol $<1.03 \mathrm{mmol} / \mathrm{l}$ in men and $<1.29 \mathrm{mmol} / \mathrm{l}$ in women or specific treatment for this lipid abnormality; (3) systolic blood pressure $\geq 130 \mathrm{mmHg}$ or diastolic blood pressure $\geq 85 \mathrm{mmHg}$ or treatment for previously diagnosed hypertension; and (4) fasting plasma glucose $\geq 5.6 \mathrm{mmol} / \mathrm{l}$ or previously diagnosed type 2 diabetes.

Analytical procedures, calculations and other measurements Plasma glucose concentrations were measured in duplicate with the glucose oxidase method using a Glucose Analyzer II (Beckman Instruments, Fullerton, CA, USA) [28]. Serum free insulin concentrations were measured with the Auto-DELFIA kit (Wallac, Turku, Finland) and Cpeptide concentrations by radioimmunoassay [29]. Serum HDL-cholesterol and triacylglycerol concentrations were measured with enzymatic kits from Roche Diagnostics using an autoanalyser (Roche Diagnostics Hitachi, Hitachi, Tokyo, Japan). Serum ALT and AST activities were determined as recommended by the European Committee for Clinical Laboratory Standards. Serum NEFA concentrations were measured using a fluorometric method [30], and serum adiponectin concentrations were measured using an ELISA kit from B-Bridge International (San Jose, CA, USA).

Glucose specific activity was determined as previously described [31]. Glucose $R_{\mathrm{a}}$ and $R_{\mathrm{d}}$ were calculated using the Steele equation, assuming a pool fraction of 0.65 for glucose and a distribution volume of $200 \mathrm{ml} / \mathrm{kg}$ for glucose [32]. The endogenous glucose $R_{\mathrm{a}}$ was calculated by subtracting the exogenous glucose infusion rate required to maintain euglycaemia during hyperinsulinaemia from the rate of total glucose $R_{\mathrm{a}}$.

The percent body fat was determined using a bioelectrical impedance analysis system (model BIA-101A; RJL Systems, Detroit, MI, USA) [33]. Waist circumference was measured midway between spina iliaca superior and the lower rib margin, and hip circumference at the level of the greater trochanters [34].

Statistical analyses The participants were divided into two equally sized groups based on their median IMCL $(9.0 \%)$ or liver fat content (3.0\%). Non-normally distributed data were used after logarithmic transformation. If distributed normally, data are shown as means \pm SEM, whereas nonnormally distributed data are shown as median $(25 \%$ percentile, $75 \%$ percentile). The unpaired Student's $t$ test was used to compare mean values between groups. Analysis of covariance was used to adjust for BMI, percent body fat, liver fat and IMCL. Correlation analyses were performed using Spearman's nonparametric rank correlation coefficient. Multiple linear regression analysis was used to analyse independent determinants of liver fat content and muscle insulin sensitivity.

Calculations were made using GraphPad Prism version 4.00 for Windows (GraphPad Software, San Diego, CA, USA), SysStat Statistical Package (SysStat version 10;
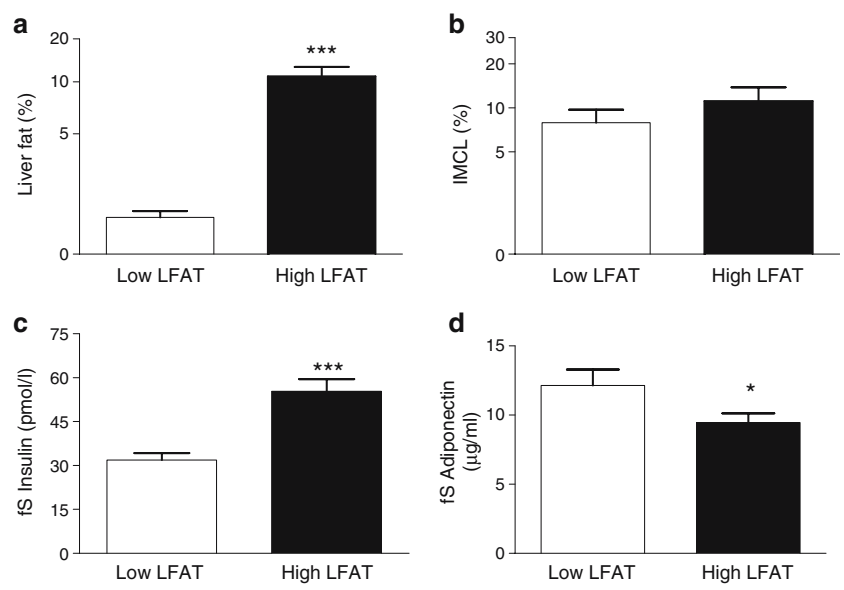

Fig. 1 Bar graphs showing liver fat (log scale) (a), IMCL (log scale) (b), fasting serum insulin (c) and fasting serum adiponectin (d) in the low and high liver fat groups. $* p \leq 0.05 ; * * * p<0.0001$ vs the corresponding low fat group. IS, fasting serum; LFAT, liver fat 
Table 1 Subject characteristics

\begin{tabular}{|c|c|c|c|c|}
\hline & Low liver fat & High liver fat & Low IMCL & High IMCL \\
\hline$n$ & 23 & 22 & 23 & 22 \\
\hline Age (years) & $43 \pm 2$ & $41 \pm 2$ & $42 \pm 2$ & $43 \pm 2$ \\
\hline \multicolumn{5}{|l|}{ Body composition } \\
\hline BMI $\left(\mathrm{kg} / \mathrm{m}^{2}\right)$ & $24.7 \pm 0.6$ & $27.7 \pm 0.9^{*}$ & $24.7 \pm 0.7$ & $27.7 \pm 0.8 * *$ \\
\hline Waist $(\mathrm{cm})$ & $91 \pm 2$ & $99 \pm 2 *$ & $91 \pm 2$ & $99 \pm 2 * *$ \\
\hline Per cent fat & $18.6 \pm 1.0$ & $22.6 \pm 1.0^{*}$ & $18.8 \pm 1.1$ & $22.4 \pm 1.0^{*}$ \\
\hline IA fat $\left(\mathrm{cm}^{3}\right)$ & $1100(650-1320)$ & $1630(930-2430)^{*}$ & $1010(680-1320)$ & $1780(1180-2600)^{* *}$ \\
\hline s.c. fat $\left(\mathrm{cm}^{3}\right)$ & $2080(1470-2910)$ & $2600(1990-3370)$ & $2000(1370-2860)$ & $2490(2010-3110)$ \\
\hline Liver fat $(\%)$ & $2.0(1.0-2.0)$ & $12.0(5.7-18.5)^{* * *}$ & $2.5(2.0-6.0)$ & $5.3(1.7-17.5)$ \\
\hline IMCL (\%) & $7.5(3.0-16.2)$ & $15.5(5.0-23.5)$ & $5.0(2.3-7.5)$ & $19.5(16.0-26.0)^{* * *}$ \\
\hline \multicolumn{5}{|l|}{ MS components } \\
\hline fP glucose (mmol/l) & $5.5 \pm 0.09$ & $5.7 \pm 0.09$ & $5.5 \pm 0.09$ & $5.6 \pm 0.12$ \\
\hline Serum triacylglycerols $(\mathrm{mmol} / \mathrm{l})$ & $1.0 \pm 0.08$ & $1.6 \pm 0.2 * *$ & $1.12 \pm 0.09$ & $1.50 \pm 0.22$ \\
\hline Serum HDL-cholesterol (mmol/l) & $1.48 \pm 0.07$ & $1.26 \pm 0.06^{*}$ & $1.38 \pm 0.06$ & $1.36 \pm 0.08$ \\
\hline Systolic BP (mmHg) & $130 \pm 3$ & $133 \pm 3$ & $128 \pm 3$ & $136 \pm 4$ \\
\hline Diastolic BP (mmHg) & $85 \pm 2$ & $87 \pm 3$ & $83 \pm 2$ & $89 \pm 2$ \\
\hline \multicolumn{5}{|l|}{ Other parameters } \\
\hline fS insulin (pmol/l) & $32 \pm 2$ & $55 \pm 4 * * *$ & $39 \pm 3$ & $48 \pm 5$ \\
\hline $\mathrm{fS}$ adiponectin $(\mu \mathrm{g} / \mathrm{ml})$ & $12.2 \pm 1.2$ & $9.5 \pm 1.2^{*}$ & $11.4 \pm 0.9$ & $10.3 \pm 1.0$ \\
\hline fS NEFA (ìmol/1) & $556 \pm 39$ & $647 \pm 45$ & $551 \pm 33$ & $643 \pm 49$ \\
\hline Serum ALT (U/1) & $26 \pm 2$ & $48 \pm 4 * * *$ & $31 \pm 3$ & $43 \pm 5$ \\
\hline Serum AST (U/l) & $26 \pm 2$ & $35 \pm 2 * * *$ & $28 \pm 2$ & $33 \pm 3$ \\
\hline$M$ value $\left[1 \mu \mathrm{mol}(\mathrm{kg} \mathrm{FFM})^{-1} \min ^{-1}\right]$ & $34.4 \pm 2.1$ & $23.0 \pm 2.1 * * *$ & $31.5 \pm 2.4$ & $26.0 \pm 2.4$ \\
\hline$\dot{V} \mathrm{O}_{2 \max }\left[\mathrm{ml}(\mathrm{kg} \mathrm{FFM})^{-1} \min ^{-1}\right]$ & $45.2 \pm 1.2$ & $41.1 \pm 1.4^{*}$ & $44.3 \pm 1.4$ & $42.0 \pm 1.3$ \\
\hline
\end{tabular}

Data are shown as means \pm SEM or median (25th percentile, 75 th percentile)

${ }^{*} p \leq 0.05 ; * * p<0.01 ; * * * p<0.0001$ vs corresponding low fat group

$\mathrm{fP}$, fasting plasma; fS, fasting serum; IA, intra-abdominal; MS, metabolic syndrome

SysStat, Evanston, IL, USA) and SPSS 11.0 for Windows (SPSS, Chicago, IL, USA). A $p$ value of less than 0.05 was considered statistically significant.

\section{Results}

Characteristics of the study groups The high and low liver fat groups were similar with respect to age. The high liver fat group was more obese than the low liver fat group, but the groups did not differ with respect to IMCL (Fig. 1). Fasting serum insulin and liver enzymes were higher and fasting serum adiponectin lower in the high than in the low liver fat group (Table 1, Fig. 1). The differences in fasting serum insulin $(p<0.0001)$, serum ALT $(p<0.0001)$ and serum AST $(p=0.001)$ remained significant after adjusting for BMI. Fasting serum adiponectin concentrations did not differ after adjusting for percent body fat $(p=0.09)$.

The low and high IMCL groups were similar with respect to age, but the high IMCL group was more obese than the low one (Table 1). Liver fat was comparable between the groups, as were fasting serum insulin and fasting serum adiponectin concentrations (Fig. 2). BMI was similar between the low liver fat and low IMCL groups, and between the high liver fat and high IMCL groups (Table 1). IMCL was not correlated with liver fat content $(r=0.16)$.
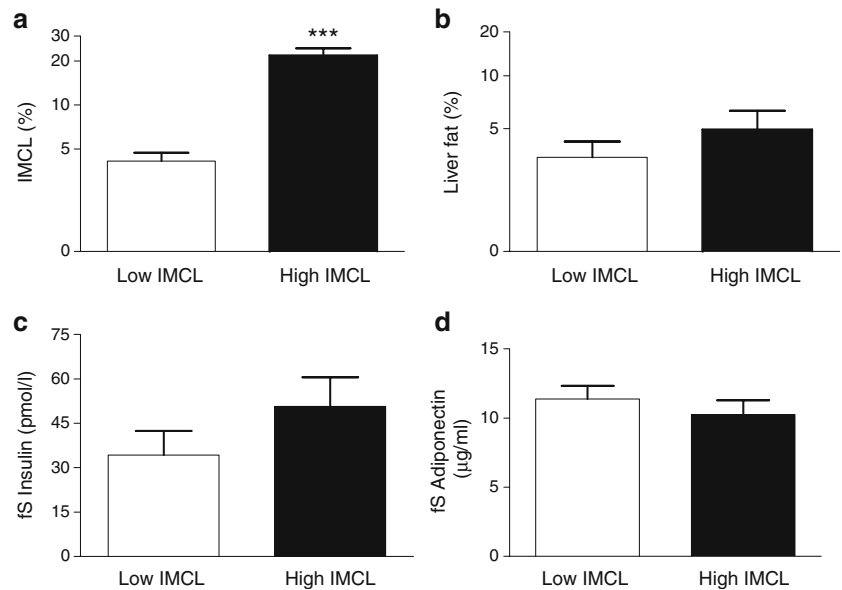

Fig. 2 Bar graphs showing IMCL (log scale) (a), liver fat (log scale) (b), fasting serum insulin (c) and fasting serum adiponectin (d) in the low and high IMCL groups. ${ }^{* * *} p<0.0001$ vs the low IMCL group. $\mathrm{fS}$, fasting serum 
Components of the metabolic syndrome In the high liver fat group, serum triacylglycerols were higher and serum HDLcholesterol concentrations lower than in the low liver fat group (Table 1). The groups were comparable with respect to fasting plasma glucose and systolic and diastolic blood pressure. These results remained unchanged if adjusted for IMCL. There was no significant difference in any component of the metabolic syndrome between the high and low IMCL groups (Table 1) even after adjusting for liver fat content. In participants with the metabolic syndrome $(n=$ $14)$, liver fat was higher than in those without the syndrome $(n=31)$ even independently of BMI $(p<0.05)$ and IMCL $(p=0.003)$, whereas IMCL was comparable between the participants with and without the metabolic syndrome (Fig. 3), even if adjusted for liver fat.

Hepatic and muscle insulin sensitivity Rates of basal endogenous glucose $R_{\mathrm{a}}$ were comparable between the high and the low liver fat groups $[14.7 \pm 0.7$ and $13.6 \pm 0.6 \mu \mathrm{mol}$ (kg FFM) ${ }^{-1} \min ^{-1}$, respectively]. During the last hour of the insulin infusion, the percent suppression of endogenous glucose $R_{\mathrm{a}}$ was inversely related to liver fat content ( $r=$ $-0.30, p<0.05$; Fig. 4) and averaged 79\% (51-120\%) vs 97\% (75-129\%; high vs low liver fat group, $p<0.05)$. Muscle insulin sensitivity ( $M$ value) was significantly lower in the high $\left[23.0 \pm 2.1 \mu \mathrm{mol}(\mathrm{kg} \mathrm{FFM})^{-1} \mathrm{~min}^{-1}\right]$ than in the low $\left[34.4 \pm 2.1 \mu \mathrm{mol}(\mathrm{kg} \mathrm{FFM})^{-1} \min ^{-1}, p=0.0008\right]$ liver fat group. In multiple linear regression analysis, $\mathrm{BMI}(p=$ $0.016)$ but not $\dot{V} \mathrm{O}_{2 \max }(p=0.19)$ independently explained variation in liver fat $\left(r^{2}=21 \%, p=0.007\right.$ for ANOVA).

The low and high IMCL groups were comparable with respect to basal endogenous glucose $R_{\mathrm{a}}$ in the basal state $\left[14.4 \pm 0.7\right.$ vs $14.0 \pm 0.6 \mu \mathrm{mol}(\mathrm{kg} \mathrm{FFM})^{-1} \mathrm{~min}^{-1}$, respectively], with no difference in percent suppression [96 (64$145)$ vs $84 \%(56-109 \%)]$ or muscle insulin sensitivity $\left[26.0 \pm 2.4\right.$ vs $31.5 \pm 2.4 \mu \mathrm{mol}(\mathrm{kg} \mathrm{FFM})^{-1} \mathrm{~min}^{-1}$, high vs low IMCL group] between the IMCL groups.

In simple linear regression analysis, muscle insulin sensitivity was inversely related to BMI $(r=-0.57, p<$ $0.0001 ; r=-0.50, p<0.0001$ if adjusted for liver fat) and positively related to $\dot{V} \mathrm{O}_{2 \max }(r=0.41, p=0.005 ; r=-0.18$, if adjusted for liver fat; Fig. 5). In multiple linear regression
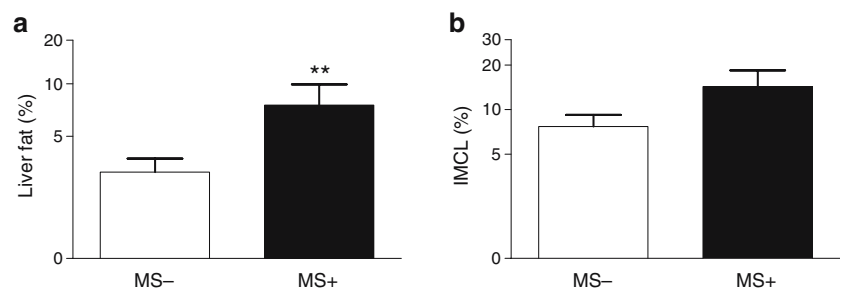

Fig. 3 Bar graphs (log scale) showing liver fat (a) and IMCL (b) in participants without $(-)$ and with $(+)$ the metabolic syndrome (MS). ${ }^{* *} p<0.01$ vs individuals without the metabolic syndrome

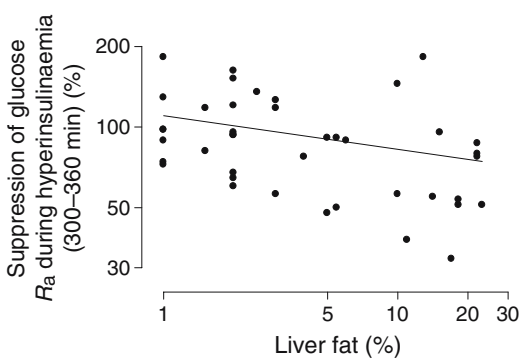

Fig. 4 Graph showing the relationship between percentage suppression of endogenous glucose production during the last hour of hyperinsulinaemia (300-360 min, log scale) and liver fat content (log scale) in individual participants (circles). $r=-0.30, p<0.05$

analysis, BMI $(p<0.0001)$ and $\dot{V} \mathrm{O}_{2 \max }(p<0.05)$ were independent determinants of muscle insulin sensitivity and together explained $46.7 \%$ of its variation $(p<0.0001$ for ANOVA). If liver fat was incorporated into these analyses, BMI $(p<0.0001)$ and liver fat $(p=0.035)$, but not $\dot{V} \mathrm{O}_{2 \max }$ $(p=0.12)$, were independent determinants of muscle insulin sensitivity $\left(r^{2}=52 \%, p<0.0001\right.$ for ANOVA). IMCL correlated significantly with BMI $(r=0.47, p=0.001 ; r=0.35, p=$ 0.018 after adjusting for liver fat), but was not related to $\dot{V} \mathrm{O}_{2 \text { max }}(r=-0.09 ; r=-0.05$ adjusted for liver fat) or muscle insulin sensitivity $(r=-0.18 ; r=-0.11$ adjusted for liver fat; Fig. 5).

Serum NEFA concentrations during prolonged low-dose insulin infusion In the liver fat groups, fasting serum NEFA concentrations were comparable in the basal state, but after 30 min of the $1.8 \mathrm{pmol} \mathrm{kg}^{-1} \mathrm{~min}^{-1}$ insulin infusion, serum NEFA concentrations were significantly higher in the high than in the low liver fat group $(459 \pm 30$ vs $354 \pm 25 \mu \mathrm{mol} / 1$, respectively, $p=0.009$ ). During the next $3.5 \mathrm{~h}$ of insulin infusion, serum NEFA remained significantly higher in the high than in the low liver fat group (Fig. 6). The difference in mean serum NEFA remained significant after adjusting for age and BMI $(p=0.03)$. In the IMCL groups, fasting serum NEFA concentrations were similar basally and during the first $2 \mathrm{~h}$ of insulin infusion. During the last hour, serum NEFA concentrations were significantly higher in the high IMCL group (Fig. 6), but after adjusting for BMI, these differences did not remain significant.

\section{Discussion}

In the present study, liver fat was fivefold higher in participants with than in those without the metabolic syndrome; this finding was independent of obesity. No differences in IMCL content could be observed between those with and those without the syndrome. The fatty liver was shown to be insulin-resistant when measured directly. These data suggest that in the present study group, 
Fig. 5 Graphs showing the relationships between (a) BMI and IMCL (log scale; $r=0.47$, $p=0.001$ ); (b) $M$ value and IMCL (log scale; $r=-0.18$ ); (c) $\dot{V} \mathrm{O}_{2 \max }$ and IMCL (log scale; $r=-0.09$ ); (d) $M$ value and $\dot{V} \mathrm{O}_{2 \max } \quad(r=0.41$, $p=0.005$ ); (e) $\mathrm{BMI}$ and $\dot{V} \mathrm{O}_{2 \max }$ ( $r=-0.30, p=0.044)$; (f) $M$ value (log scale) and BMI $(r=-0.57$, $p<0.0001$ ); and (g) $M$ value and liver fat content $(\log$ scale; $r=-0.52, p=0.0002$ ) a

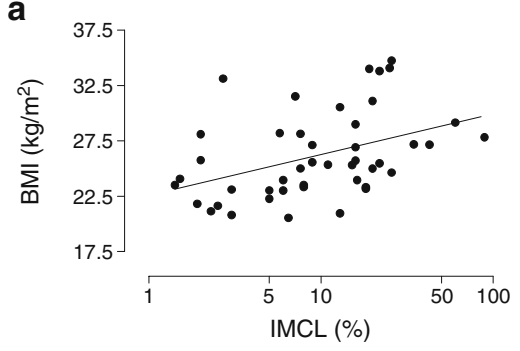

b

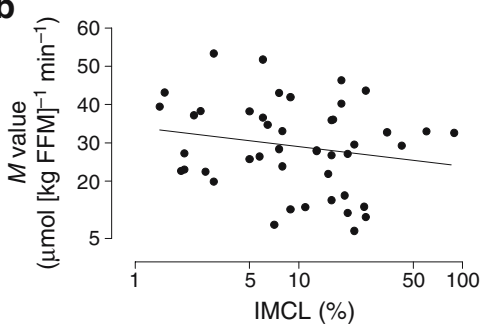

C

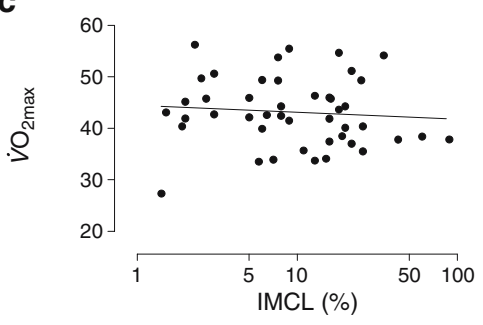

d

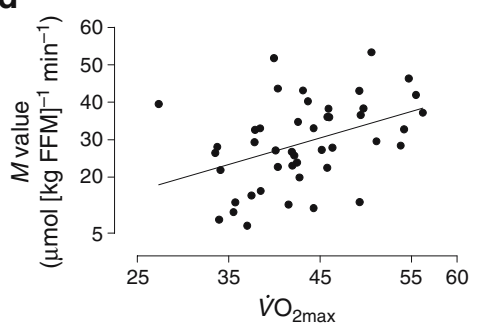

e

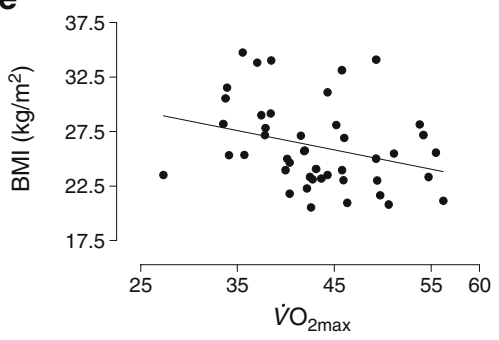

f

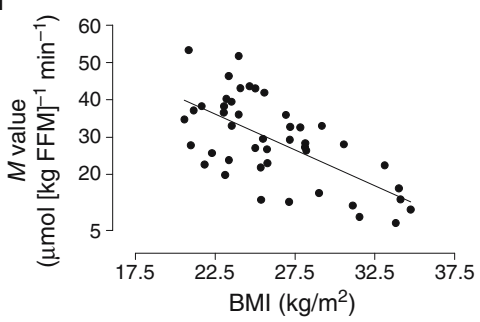

g

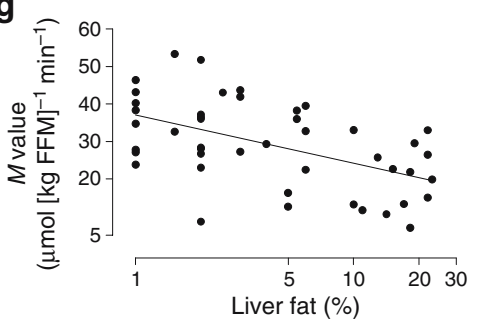

intrahepato- rather than IMCL accumulation was a marker of the metabolic syndrome, i.e. increased serum triacylglycerols and decreased HDL-cholesterol, as well as hyperinsulinaemia and low adiponectin.

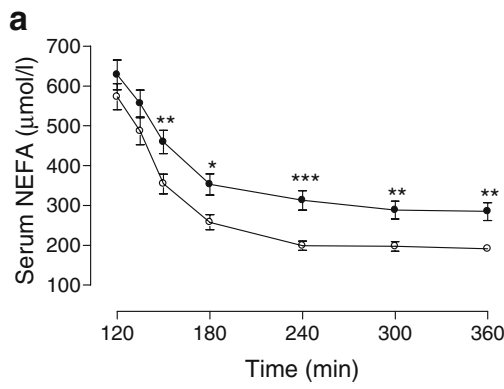

Fig. 6 Serum NEFA concentrations during the low-dose insulin infusion (120-360 min) in the high (black circles) and low (white circles) liver fat groups (a) and in the high (black circles) and low (white circles) IMCL groups (b). The NEFA concentrations were
Consistent with previous data, we found obesity to be associated with increased IMCL [12, 15], decreased $\dot{V} \mathrm{O}_{2 \max }$ [35] and whole-body insulin sensitivity [36] (Fig. 5). Since IMCL is increased by enhanced physical

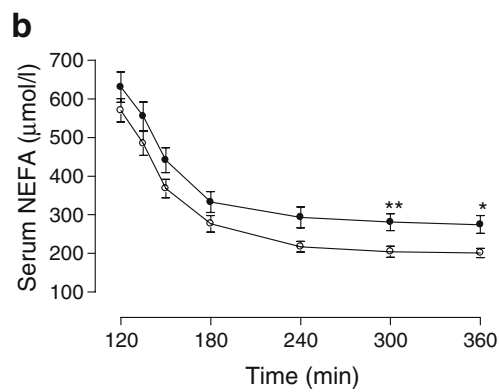

compared using ANOVA for repeated measures followed Tukey's 'honestly significant differences' test for pairwise comparisons. ${ }^{*} p \leq$ $0.05 ; * * p<0.01 ; * * * p<0.0001$ 
fitness [12-14] and obesity [12, 15], the lack of a relationship between IMCL and muscle insulin sensitivity is not surprising. As in the present study, Thamer et al. found neither $\dot{V} \mathrm{O}_{2 \max }$ nor insulin sensitivity to be associated with IMCL [12]. However, further analyses revealed a significant positive association between $\dot{V} \mathrm{O}_{2}$ max and IMCL in lean, but not in obese individuals [12]. In the present study, IMCL was not related to $\dot{V} \mathrm{O}_{2 \max }$, possibly because the study participants covered a wide range of obesity, with none participating in regular physical exercise. Nevertheless, BMI was inversely related to $\dot{V} \mathrm{O}_{2}$ max expressed per FFM (Fig. 5). This may explain why IMCL was not inversely related to muscle insulin sensitivity, although $\dot{V} \mathrm{O}_{2}$ max was an independent determinant of muscle insulin sensitivity (Fig. 5).

Hepatic fat content has been shown to associate with insulin resistance of hepatic glucose production [5, 6, 37]. We now extended these findings to show that liver fat is associated with hepatic insulin resistance in the absence of changes in IMCL and that liver fat and IMCL were not correlated. This implies that sites of intracellular triacylglycerol accumulation exhibit interindividual variation. It is noteworthy that although liver fat is a marker of hepatic insulin resistance, it is highly unlikely that the metabolically inert triacylglycerols mediate the impairment in insulin action [38, 39]. Lipid intermediates that increase as a function of intracellular lipid stores, such as diacylglycerol [40] or ceramides [41], are likely to be responsible.

Regarding the reasons why liver fat is associated with features of the metabolic syndrome, we have previously shown that failure of insulin to suppress hepatic VLDL production results in hypertriacylglycerolaemia and a low HDL-cholesterol concentration [42], and that VLDL overproduction rather than impaired clearance correlates with liver fat content [7]. Resistance to insulin suppression of triacylglycerol production, although not measured in this study, is therefore a likely explanation of the finding that serum triacylglycerols were higher and HDL-cholesterol lower in the high as compared with the low liver fat groups.

Intrahepatic fat content is frequently referred to as an additional feature of the metabolic syndrome [43]. In the present study, liver fat was fivefold higher in participants with than in those without the metabolic syndrome, a finding that was independent of obesity. On the other hand, no differences in IMCL content could be observed between participants with and without the metabolic syndrome. Our findings suggest that depots of lipid accumulation in the liver and skeletal muscle do not contribute in an interactive manner to the metabolic syndrome.

In the present study, fasting serum NEFA concentrations were not associated with liver fat or IMCL content, but they remained significantly higher during the low-dose insulin infusion in participants with high liver fat content, indepen- dently of obesity. This finding is in keeping with previous studies in patients with non-alcoholic fatty liver disease as compared with weight-matched healthy controls [44]. The reason for the lack of any relationship between fasting serum NEFA and liver fat content is unclear. Possibly, higher NEFA during hyperinsulinaemia could reflect insulin resistance of lipolysis [43]. In keeping with differential regulation of serum NEFA basally and during hyperinsulinaemia, basal lipolysis is unaltered in hormone sensitive lipase-null mice [45]. There are, however, no human data describing alterations in NEFA fluxes across the splanchnic bed and therefore alterations in NEFA utilisation in individuals with high vs low liver fat content cannot be ruled out.

We conclude that intrahepatocellular rather than intramyocellular fat associates with the metabolic syndrome, characterised by increased triacylglycerol and decreased HDL-cholesterol concentrations, independently of obesity. This may in part be because IMCL is not correlated with muscle insulin sensitivity in individuals whose muscle insulin sensitivity is regulated by both obesity and physical fitness, as in the present study and that of Thamer et al. [12]. The data thus suggest that ectopic fat deposition in the liver may be a useful additional marker of the metabolic syndrome. In this respect, the identification of novel circulating markers suitable for use in the clinic as measures of liver fat would be helpful.

Acknowledgements This study was supported by research grants from the Academy of Finland, the Sigrid Juselius Foundation, the Medical Society of Finland and Novo Nordisk Foundation. This work is part of the project 'Hepatic and adipose tissue and functions in the metabolic syndrome' (http://www.hepadip.org), which is supported by the European Commission as an Integrated Project under the 6th Framework Programme (Contract LSHM-CT-2005-018734).

We gratefully acknowledge $\mathrm{K}$. Tuominen for excellent technical assistance, A.-M. Häkkinen for her contribution to analysing spectroscopy data and the volunteers for their help.

Duality of interest The authors declare that there is no duality of interest associated with this manuscript.

\section{References}

1. Alberti KG, Zimmet P, Shaw J, IDF Epidemiology Task Force Consensus Group (2005) The metabolic syndrome-a new worldwide definition. Lancet 366:1059-1062

2. Reaven GM (1988) Banting lecture 1988. Role of insulin resistance in human disease. Diabetes 37:1595-1607

3. Yki-Jarvinen H (1993) Action of insulin on glucose metabolism in vivo. Baillieres Clin Endocrinol Metab 7:903-927

4. Malmstrom R, Packard CJ, Watson TD et al (1997) Metabolic basis of hypotriglyceridemic effects of insulin in normal men. Arterioscler Thromb Vasc Biol 17:1454-1464

5. Ryysy L, Hakkinen AM, Goto T et al (2000) Hepatic fat content and insulin action on free fatty acids and glucose metabolism 
rather than insulin absorption are associated with insulin requirements during insulin therapy in type 2 diabetic patients. Diabetes 49:749-758

6. Seppala-Lindroos A, Vehkavaara S, Hakkinen AM et al (2002) Fat accumulation in the liver is associated with defects in insulin suppression of glucose production and serum free fatty acids independent of obesity in normal men. J Clin Endocrinol Metab 87:3023-3028

7. Adiels M, Taskinen MR, Packard C et al (2006) Overproduction of large VLDL particles is driven by increased liver fat content in man. Diabetologia 49:755-765

8. Kadowaki T, Yamauchi T (2005) Adiponectin and adiponectin receptors. Endocr Rev 26:439-451

9. Krssak M, Falk Petersen K, Dresner A et al (1999) Intramyocellular lipid concentrations are correlated with insulin sensitivity in humans: a 1H NMR spectroscopy study. Diabetologia 42:113-116

10. Jacob S, Machann J, Rett K et al (1999) Association of increased intramyocellular lipid content with insulin resistance in lean nondiabetic offspring of type 2 diabetic subjects. Diabetes 48:1113-1119

11. Pan DA, Lillioja S, Kriketos AD et al (1997) Skeletal muscle triglyceride levels are inversely related to insulin action. Diabetes 46:983-988

12. Thamer C, Machann J, Bachmann O et al (2003) Intramyocellular lipids: anthropometric determinants and relationships with maximal aerobic capacity and insulin sensitivity. J Clin Endocrinol Metab 88:1785-1791

13. Hurley BF, Nemeth PM, Martin WH, 3rd, Hagberg JM, Dalsky GP, Holloszy JO (1986) Muscle triglyceride utilization during exercise: effect of training. J Appl Physiol 60:562-567

14. van Loon LJ, Goodpaster BH (2006) Increased intramuscular lipid storage in the insulin-resistant and endurance-trained state. Pflugers Arch 451:606-616

15. Goodpaster BH, Theriault R, Watkins SC, Kelley DE (2000) Intramuscular lipid content is increased in obesity and decreased by weight loss. Metabolism 49:467-472

16. Krssak M, Petersen KF, Bergeron R et al (2000) Intramuscular glycogen and intramyocellular lipid utilization during prolonged exercise and recovery in man: a $13 \mathrm{C}$ and $1 \mathrm{H}$ nuclear magnetic resonance spectroscopy study. J Clin Endocrinol Metab 85:748-754

17. Pruchnic R, Katsiaras A, He J, Kelley DE, Winters C, Goodpaster BH (2004) Exercise training increases intramyocellular lipid and oxidative capacity in older adults. Am J Physiol Endocrinol Metab 287:E857-E862

18. Yki-Jarvinen H, Young AA, Lamkin C, Foley JE (1987) Kinetics of glucose disposal in whole body and across the forearm in man. J Clin Invest 79:1713-1719

19. DeFronzo RA, Tobin JD, Andres R (1979) Glucose clamp technique: a method for quantifying insulin secretion and resistance. Am J Physiol 237:E214-E223

20. Rizza RA, Mandarino LJ, Gerich JE (1981) Dose-response characteristics for effects of insulin on production and utilization of glucose in man. Am J Physiol 240:E630-E639

21. Ferrannini E, Mari A (1998) How to measure insulin sensitivity. J Hypertens 16:895-906

22. DeFronzo RA, Jacot E, Jequier E, Maeder E, Wahren J, Felber JP (1981) The effect of insulin on the disposal of intravenous glucose. Results from indirect calorimetry and hepatic and femoral venous catheterization. Diabetes 30:1000-1007

23. Thomsen C, Becker U, Winkler K, Christoffersen P, Jensen M, Henriksen O (1994) Quantification of liver fat using magnetic resonance spectroscopy. Magn Reson Imaging 12:487-495

24. Virkamaki A, Korsheninnikova E, Seppala-Lindroos A et al (2001) Intramyocellular lipid is associated with resistance to in vivo insulin actions on glucose uptake, antilipolysis, and early insulin signaling pathways in human skeletal muscle. Diabetes 50:2337-2343

25. Boesch C, Kreis R (2000) Observation of intramyocellular lipids by 1H-magnetic resonance spectroscopy. Ann N Y Acad Sci 904:25-31

26. Schick F, Eismann B, Jung WI, Bongers H, Bunse M, Lutz O (1993) Comparison of localized proton NMR signals of skeletal muscle and fat tissue in vivo: two lipid compartments in muscle tissue. Magn Reson Med 29:158-167

27. Szczepaniak LS, Babcock EE, Schick F et al (1999) Measurement of intracellular triglyceride stores by $\mathrm{H}$ spectroscopy: validation in vivo. Am J Physiol 276:E977-E989

28. Kadish AH, Little RL, Sternberg JC (1968) A new and rapid method for the determination of glucose by measurement of rate of oxygen consumption. Clin Chem 14:116-131

29. Kuzuya H, Blix PM, Horwitz DL, Steiner DF, Rubenstein AH (1977) Determination of free and total insulin and C-peptide in insulin-treated diabetics. Diabetes 26:22-29

30. Miles J, Glasscock R, Aikens J, Gerich J, Haymond M (1983) A microfluorometric method for the determination of free fatty acids in plasma. J Lipid Res 24:96-99

31. Puhakainen I, Koivisto VA, Yki-Jarvinen H (1991) No reduction in total hepatic glucose output by inhibition of gluconeogenesis with ethanol in NIDDM patients. Diabetes 40:1319-1327

32. Steele R (1959) Influences of glucose loading and of injected insulin on hepatic glucose output. Ann N Y Acad Sci 82:420-430

33. Lukaski HC, Johnson PE, Bolonchuk WW, Lykken GI (1985) Assessment of fat-free mass using bioelectrical impedance measurements of the human body. Am J Clin Nutr 41:810-817

34. Marti B, Tuomilehto J, Salomaa V, Kartovaara L, Korhonen HJ, Pietinen P (1991) Body fat distribution in the Finnish population: environmental determinants and predictive power for cardiovascular risk factor levels. J Epidemiol Community Health 45:131137

35. Coon PJ, Rogus EM, Drinkwater D, Muller DC, Goldberg AP (1992) Role of body fat distribution in the decline in insulin sensitivity and glucose tolerance with age. J Clin Endocrinol Metab 75:1125-1132

36. Yki-Jarvinen H, Koivisto VA (1983) Effects of body composition on insulin sensitivity. Diabetes 32:965-969

37. Marchesini G, Brizi M, Bianchi G et al (2001) Nonalcoholic fatty liver disease: a feature of the metabolic syndrome. Diabetes 50:1844-1850

38. Morino K, Petersen KF, Shulman GI (2006) Molecular mechanisms of insulin resistance in humans and their potential links with mitochondrial dysfunction. Diabetes 55(Suppl 2):S9-S15

39. Summers SA (2006) Ceramides in insulin resistance and lipotoxicity. Prog Lipid Res 45:42-72

40. Nagle CA, An J, Shiota M et al (2007) Hepatic overexpression of glycerol-sn-3-phosphate acyltransferase 1 in rats causes insulin resistance. J Biol Chem 282:14807-14815

41. Holland WL, Brozinick JT, Wang LP et al (2007) Inhibition of ceramide synthesis ameliorates glucocorticoid-, saturated-fat-, and obesity-induced insulin resistance. Cell Metab 5:167-179

42. Malmstrom R, Packard CJ, Caslake M et al (1997) Defective regulation of triglyceride metabolism by insulin in the liver in NIDDM. Diabetologia 40:454-462

43. Utzschneider KM, Kahn SE (2006) The role of insulin resistance in non-alcoholic fatty liver disease. J Clin Endocrinol Metab 91:4753-4761

44. Bugianesi E, Gastaldelli A, Vanni E et al (2005) Insulin resistance in non-diabetic patients with non-alcoholic fatty liver disease: sites and mechanisms. Diabetologia 48:634-642

45. Wang SP, Laurin N, Himms-Hagen J et al (2001) The adipose tissue phenotype of hormone-sensitive lipase deficiency in mice. Obes Res 9:119-128 